\title{
Impact of Regions with COVID-19 Cases on COVID-Zero Regions by Population Mobility - Worldwide, 2021
}

\author{
Lianhao Wang'; Shiyi Wang'; Xiangyu Zhang'; Zhongwei Jia ${ }^{1,3,4,4}$
}

\section{Summary \\ What is already known about this topic?}

Reducing population mobility and increasing the vaccination rate for severe acute respiratory syndrome coronavirus 2 can decrease the transmission of coronavirus disease 2019 (COVID-19).

What is added by this report?

In order to reduce the incidence of COVID-19 to the levels of influenza after restoring normal mobility, the efficacy against infection needs to be increased to $40 \%$ and the efficacy against symptomatic disease needs to be increased to $90 \%$. The efficacy against infection has a more important impact compared to efficacy against symptomatic disease or death on the transmission of COVID-19 at the population level.

What are the implications for public health practice?

The population should continue maintaining nonpharmaceutical interventions and minimize international movement to prevent transmission of COVID-19. Furthermore, developing new vaccines or promoting booster vaccinations should be considered to increase efficacy.

The development of vaccines has made a great contribution to the fight against coronavirus disease 2019 (COVID-19), but it should be clearly recognized that the elimination of severe acute respiratory syndrome coronavirus 2 (SARS-CoV-2) requires the combined efforts of humanity. In order to determine how the population mobility among different regions might affect the COVID-19 epidemic, we constructed a transregional mobility dynamics model to assess the relationship between population mobility and the COVID-19 pandemic. Based on a previous study on vaccine efficacy against infection in the United Kingdom ( 1 ) and a study in Chile on the vaccine protective effects of CoronaVac (2), the baseline efficacy, indicated by efficacy against infection $\left(\mathrm{E}_{\text {inf }}\right)$, efficacy against symptomatic disease $\left(\mathrm{E}_{\text {sym }}\right)$, and efficacy against death $\left(\mathrm{E}_{\text {dea }}\right)$ was respectively $30 \%, 68.3 \%$, and
$86 \%$. If the vaccination rate reaches $95 \%$, allowing for transregional movement would result in 234.2 million infections (64.1 million symptomatic cases) and 2.0 million deaths (case fatality rate of $0.85 \%$ ) within a year in unaffected regions. Increasing $\mathrm{E}_{\text {inf }}$ and $\mathrm{E}_{s y m}$ to no less than $40 \%$ and $90 \%$, respectively, could reduce the incidence of COVID-19 in COVID-zero regions to influenza-like levels. When the $\mathrm{E}_{\text {inf }}$ of the vaccine was higher, the $\mathrm{E}_{s y m}$ could be reduced accordingly. No matter how effective the vaccine was, it could not eliminate COVID-19 in COVID-zero regions, i.e., regions with strong national commitments to suppressing COVID-19 transmission such as China. The human race should continue to develop vaccines and explore new ways to improve vaccine protection against infection in order to eliminate COVID-19 at the global level.

In order to group the transmission risk of COVID-19 in various countries around the world, we used a proportion of existing cases in the overall population of countries to rank and group these countries and regions into three levels, including highrisk regions, medium-risk regions, and low-risk regions. COVID-zero regions were defined as countries or regions committed to reducing the number of domestic cases to zero, such as China. The countries covered by each region were shown in Figure 1A. Considering the disease characteristics of COVID-19, we added categories to include asymptomatic infected cases and divided each category into two according to vaccination status (Figure 1B). The model assumed that the mobility rate between COVID-zero regions and other areas was constant. All parameters used in this model and COVID-19 epidemic data sources were shown in Table 1, and more details of methods were shown in Supplementary Materials, available in http:// weekly.chinacdc.cn/.

Based on the study in Chile mentioned above, $\mathrm{E}_{\text {sym }}$ and $\mathrm{E}_{\text {dea }}$ of CoronaVac was $68.3 \%$ and $86 \%$, respectively (2). And the $\mathrm{E}_{\text {inf }}$ was estimated to $55 \%$ by the study produced in the United Kingdom (1). Considering the declining immunity in the vaccinated population, $\mathrm{E}_{\text {inf }}$ was finally estimated to $30 \%$ 

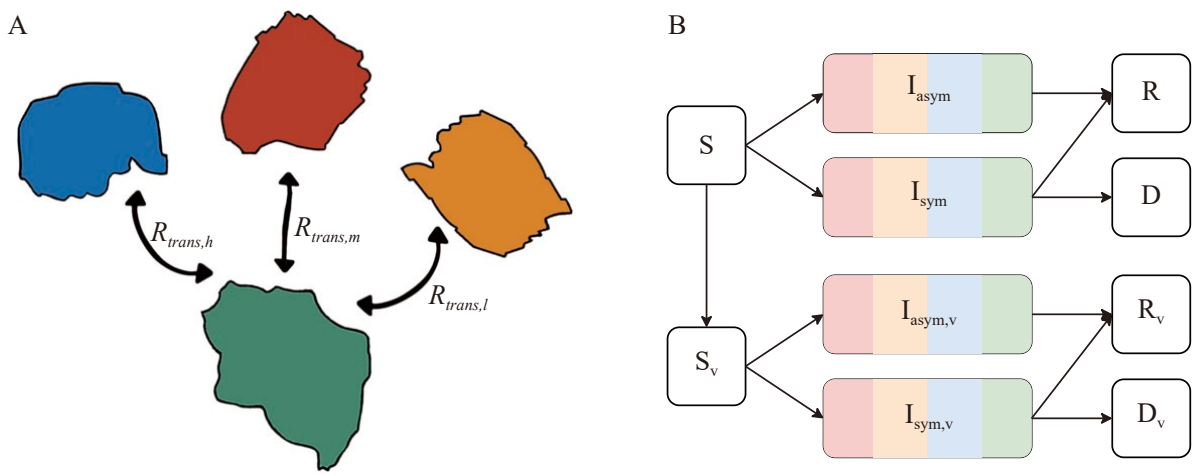

FIGURE 1. Diagrams of the COVID-19 epidemic model. (A) the diagram of transregional mobility of population, (B) epidemic dynamics model compartments.

Note: $S, I_{\text {asym }}, I_{\text {sym }}, R$, and D desperately denoted the susceptible, asymptomatic infective, symptomatic infective, removal and dead. And the v subscript indicated COVID-19 vaccination.

Abbreviation: COVID-19=coronavirus disease 2019.

TABLE 1. Estimation of COVID-19 transregional model parameters and data sources.

\begin{tabular}{ccc}
\hline Label & Value & Reference \\
\hline$R_{\text {trans }}$ & $0.0002,0.0013^{*}$ & $(3-4)$ \\
$R_{0}$ & 2.5 & $(5)$ \\
$R_{\text {sym }}$ & $70 \%$ & $(5)$ \\
$R_{\text {dea }}$ & $2.0 \%$ & Calculated $^{\dagger}$ \\
$R_{\text {rem }}$ & $1 / 5.4^{\S}$ & $(6)$ \\
$\beta$ & 0.179 & Calculated by $R_{0}$ \\
$R_{a-s}$ & $75 \%$ & $(6)$ \\
$\mathrm{E}_{\text {inf }}$ & $30 \%$ & Based on $(1) \pi$ \\
$\mathrm{E}_{\text {sym }}$ & $65.3 \%$ & $(2)$ \\
$\mathrm{E}_{\text {dea }}$ & $86 \%$ & $(2)$ \\
\hline
\end{tabular}

${ }^{*} 0.0002$ was the number of arrivals and departures of January to June 2021 and 0.0013 was from the whole year of 2019 . They represented the current population mobility rate and normal mobility rate.

${ }^{\dagger} R_{\text {dea }}$ is the sum of deaths divided by the sum of confirmed cases in all countries.

$\S R_{r e m}$ denoted that patients presented to the doctor within 2 days since the onset of symptoms (average 3.4 days) and were no longer transmissible.

"More details about the estimation of $\mathrm{R}_{\text {inf }}$ could be found in

Supplementary Materials, available in http://weekly.chinacdc.cn/.

(Supplementary Material, available in http://weekly. chinacdc.cn/). Based on the baseline efficacies of the vaccines, referred to $\mathrm{E}_{\text {inf }}, \mathrm{E}_{\text {sym }}$, and $\mathrm{E}_{\text {dea }}$ were $30 \%$, $68.3 \%$, and $86 \%$, respectively, the global vaccination rate could reach $95 \%$ and then people in other areas could move within COVID-zero regions without nonpharmaceutical interventions (NPIs). Among COVIDzero regions, a total of 234.2 million SARS-CoV-2 infections were projected to occur in one year, of which 170.1 million $(72.6 \%)$ were asymptomatic cases and 64.1 million $(27.4 \%)$ are symptomatic cases
(Figure 2A), with a total death count of 2.0 million (case fatality rate $0.85 \%$ ). The highest number of new symptomatic cases per day was estimated 376,600 , which would appear on the Day 262 after lifting the restrictions and the highest number of deaths per day was 11,400, which would appear on the Day 266 (Figure 2B) in COVID-zero regions.

On the basis of maintaining the current mobility rate, we explored what kind of combination of vaccine protective efficacy could lift the restrictions. Due to the COVID-19 vaccine having an $\mathrm{E}_{\text {dea }}$ of $86 \%$, we presented results that assumed $\mathrm{E}_{\text {dea }}$ to be $90 \%$ (Figure 3A). Predictions of other combinations of vaccine protective efficacy could be found in the Supplementary Materials (available in http://weekly.chinacdc.cn/). The results suggested that in order to reduce the annual incidence of COVID-19 to influenza, the vaccine's $\mathrm{E}_{\text {inf }}$ needed to be increased to more than $40 \%$ concurrently with an $\mathrm{E}_{\text {sym }}$ of at least $60 \%$. If the $\mathrm{E}_{i n f}$ was increased to $50 \%$, the $\mathrm{E}_{\text {sym }}$ could decrease to $0 \%$. In addition, when the 3 efficacies reached at least $90 \%$, the annual incidence of COVID-19 was decreased to $0.81 / 100,000$ population.

Finally, we assessed what protective efficacy would be required to restore the population mobility to ensure that the incidence of COVID-19 could be lower than that of influenza. After restoring the population mobility rate of 2019, a higher vaccine protective effect was required. In order to reduce the annual incidence of COVID-19 to that of influenza, the vaccine's $\mathrm{E}_{\text {inf }}$ and $\mathrm{E}_{\text {sym }}$ needed to be increased to no less than $40 \%$ and $90 \%$, respectively. With a higher $\mathrm{E}_{\text {inf }}$, the $\mathrm{E}_{\text {sym }}$ could be decreased (Figure 3B). When the $\mathrm{E}_{\text {inf }}$ was increased to $70 \%$, the $\mathrm{E}_{\text {sym }}$ could be decreased to 0 (Table 2). 
A

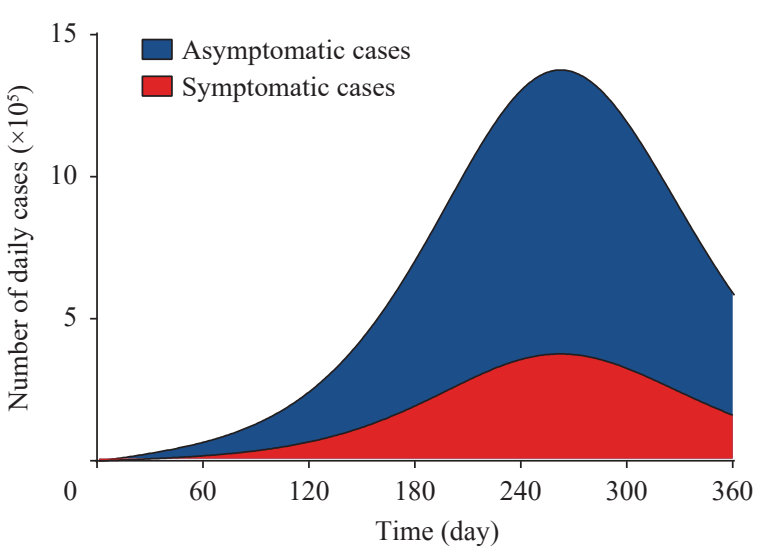

B

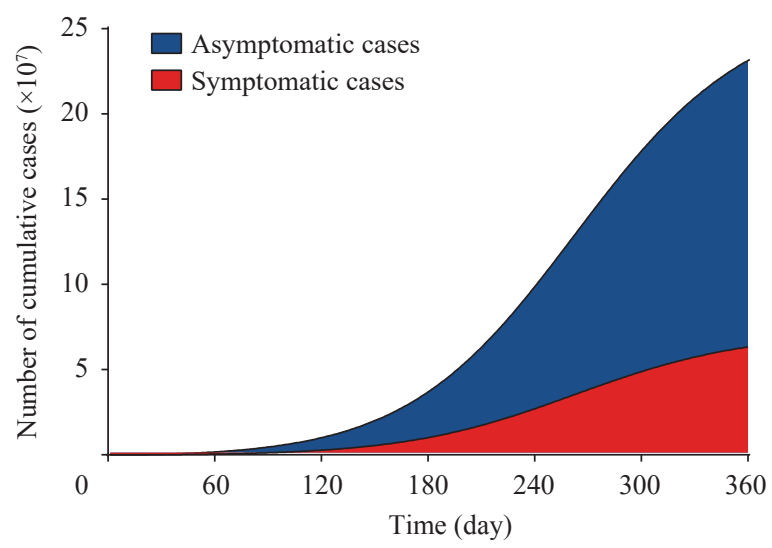

FIGURE 2. Prediction of COVID-19 epidemic after restoring population mobility without NPIs. (A) The number of daily infections, (B) The number of cumulative infections with time..

Note: Blue denoted the asymptomatic cases and red denoted the symptomatic cases. This result was based on the efficacy against infections, symptomatic disease and death was separately $30 \%, 68.3 \%$, and $86 \%$, and the vaccination rate was $95 \%$.
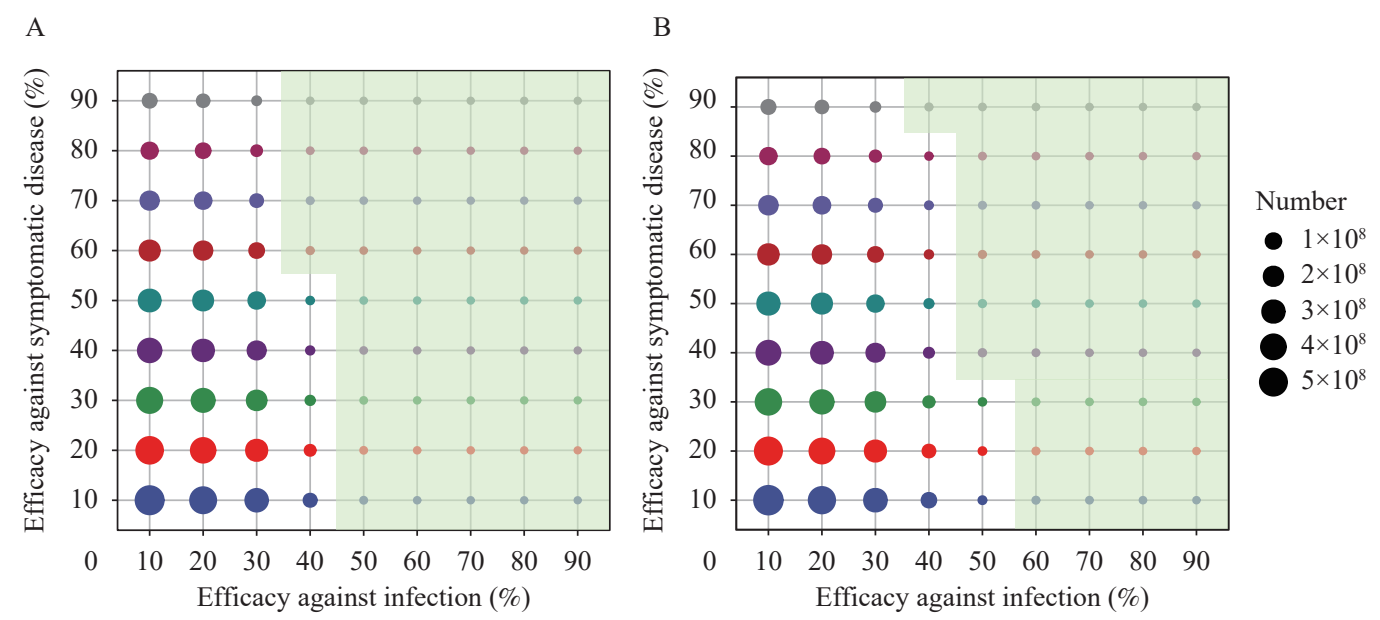

FIGURE 3. The number of COVID-19 cases in response to different vaccine protection at (A) low rate of population mobility, (B) normal rate of population mobility.

Note: The size of the circle denoted the number of COVID-19 cases and the green shadow denoted the annual incidence was lower than influenza with the corresponding efficacy against infection and symptomatic disease and a vaccination rate of $95 \%$, indicated that efficacy against death has a less impact on virus transmission so we only show the prediction of $90 \%$ efficacy against death.

TABLE 2. Prediction of COVID-19 epidemic by currently vaccine effectiveness and combinations of threshold efficacies that can reduce the number of infections to influenza.

\begin{tabular}{ccccccc}
\hline \multirow{2}{*}{$\begin{array}{c}\text { Vaccination rate } \\
(\%)\end{array}$} & Mobility rate (\%) & \multicolumn{3}{c}{ Vaccine effectiveness } & Symptomatic cases & Deaths \\
\cline { 3 - 5 } & & Infection (\%) & Symptom (\%) & Death (\%) & & \\
\hline 95 & 0.02 & 30.0 & 65.3 & 86.0 & $64,123,171$ & $2,039,690$ \\
95 & 0.02 & 40.0 & 60.0 & 90.0 & $1,054,347$ & 28,517 \\
95 & 0.02 & 50.0 & 0.0 & 90.0 & 623,887 & 12,906 \\
95 & 0.13 & 40.0 & 90.0 & 90.0 & 706,184 & 40,565 \\
95 & 0.13 & 50.0 & 40.0 & 90.0 & $1,105,650$ & 31,237 \\
95 & 0.13 & 60.0 & 0.0 & 90.0 & 627,634 & 17,808 \\
\hline
\end{tabular}

Abbreviation: COVID-19=coronavirus disease 2019.

* Indicated that efficacy against death has a less impact on virus transmission so we only showed the prediction of $90 \%$ efficacy against death. 


\section{DISCUSSION}

Our model aimed to create a hypothetical scenario in which enough people were vaccinated to assess whether people could move freely and safely without NPIs. There were three main findings of this study. First, based on the assumption of parameters that the $\mathrm{E}_{\text {inf }}, \mathrm{E}_{\text {sym }}$, and $\mathrm{E}_{\text {dea }}$ were $30 \%, 65.3 \%$, and $86 \%$, respectively, COVID-19 vaccinations were not enough to provide a sufficient proportion of immunized population to resume normal population mobility without NPIs. Among the three parameters estimating the protective effects of the vaccine, only the $\mathrm{E}_{\text {inf }}$ was supported by existing evidence. But considering immune escape ability of Delta variant, we thought estimation of $30 \%$ was reasonable. It indicated that policymakers should carefully deal with allowing transmission with freedom and all populations should continue to maintain the NPIs in order to avoid a resurgence of COVID-19.

Second, if we assumed that COVID-19 incidence was lower than that of influenza for resuming population mobility, the vaccine $\mathrm{E}_{\text {inf }}$ should be at least $40 \%$. Simply increasing the $\mathrm{E}_{s y m}$ and $\mathrm{E}_{\text {dea }}$ would not do much to control the disease. The $\mathrm{E}_{\text {inf }}$ had a more important impact on controlling COVID-19 at the population level. Therefore, further research on the efficacy against infection was of vital significance. The results of this study only assumed the minimum value of the protective effects to decrease the incidence of COVID-19 to that of influenza due to the decline in vaccine immunity and the possible emergence of new strains. In any case, resuming international population mobility should be treated with caution to avoid domestic outbreaks.

Finally, we investigated if the vaccine alone could be used to achieve a COVID-zero strategy in COVIDzero regions. Because of population mobility, even if the 3 vaccine efficacies reached 90\%, COVID-zero regions would not be able to eliminate COVID-19. This result suggested that as long as there were COVID-19 cases in other countries, vaccinations alone without non-pharmaceutical interventions could not reduce the number of domestic cases to zero. Rationally allotting the COVID-19 vaccines might play a more important role in controlling COVID-19 worldwide.

This study was subject to at least four limitations. First, the vaccine protective efficacy was assumed based on results of the Sinovac vaccine in Chile. However, due to the different types of vaccines used in various countries around the world, this may not accurately reflect results in other regions included in this study. In addition, the estimated mobility rate in this study referred to data of China in 2019 and 2021, and the mobility rate of other countries will differ significantly from that in China. Third, due to China's policy of administering booster vaccinations, the study did not consider the effect of declining immunity on vaccine effectiveness, but this may lead to further issues in predicting disease transmission. Fourth, the emergence of new SARS-CoV-2 strains might significantly increase the risk of disease transmission in a country or region, and the parameters of this model were based on the characteristics of current strains. If new variants of concern emerge, researchers need to re-model the risk of disease transmission after acquiring the information about the characteristics of new strains.

The key to controlling COVID-19 lies in the development and widespread use of vaccines that are more effective at preventing infection. People still need to maintain adequate NPIs to avoid the risk of COVID-19 recurrence.

doi: $10.46234 / \mathrm{ccdcw} 2022.012$

\#Corresponding author: Zhongwei Jia, urchinjj@163.com. ${ }^{1}$ School of Public Health, Peking University, Beijing, China; ${ }^{2}$ China
Medical Science Press, China Health Media Group, Beijing, China;
${ }_{3}$ Center for Intelligent Public Health, Institute for Artificial
Intelligence, Peking University, Beijing, China; ${ }^{4}$ Center for Drug
Abuse Control and Prevention, National Institute of Health Data
Science, Peking University, Beijing, China.

Submitted: November 12, 2021; Accepted: January 10, 2022

\section{REFERENCES}

1. Elliott P, Haw D, Wang HW, Eales O, Walters CE, Ainslie KEC, et al. Exponential growth, high prevalence of SARS-CoV-2, and vaccine effectiveness associated with the Delta variant. Science 2021;374 (6574):eabl9551. http://dx.doi.org/10.1126/science.abl9551.

2. Jara A, Undurraga EA, González C, Paredes F, Fontecilla T, Jara G, et al . Effectiveness of an inactivated SARS-CoV-2 vaccine in Chile. N Engl J Med 2021;385(10):875 - 84. http://dx.doi.org/10.1056/NEJMoa21 07715.

3. National Immigration Administration of China. Key data on immigration management in the first half of 2021. 2021. https://www. nia.gov.cn/n897453/c1431039/content.html. [2021-10-10]. (In Chinese).

4. National Immigration Administration of China. In 2019, the number of inbound and outbound visits reached 670 million. 2021. http:// www.gov.cn/xinwen/2020-02/15/content_5479396.htm. [2021-10-10]. (In Chinese).

5. U.S. Center for Disease Control and Prevention. COVID-19 pandemic planning scenarios. 2021. https://www.cdc.gov/coronavirus/2019-ncov/ hcp/planning-scenarios.html\#table-1. [2021-10-10].

6. WHO. Report of the WHO-China joint mission on coronavirus disease 2019 (COVID-19). Beijing. 2020. https://www.who.int/publications/i/ item/report-of-the-who-china-joint-mission-on-coronavirus-disease2019-(covid-19). [2021-10-10]. 


\section{SUPPLEMENTARY MATERIALS}

\section{Model Assumptions}

1. The natural growth and mortality rate of the population were not considered.

2. The mobility probability of people in the same area was consistent.

3. Recovered cases would not be reinfected with coronavirus disease 2019 (COVID-19).

4. The protective efficacy of the vaccine will not diminish during the forecast period.

5. The rates of population mobility between different regions were consistent and stable.

6. The time taken by the movement of people was ignored.

\section{Grouping Results}

We collected the COVID-19 epidemic data of 194 countries and regions except China through the COVID-19 global data of Dingxiangyuan Website (1) which included confirmed cases, recovered cases, and deaths up to October 10, 2021 and the population data collected by the World Health Organization (WHO) until October 10, 2021. The prevalence rate of COVID-19 $\left(R_{p r e, i}\right)$ in each country or region was calculated by the following methods:

$$
R_{p r e, i}=\frac{n_{a c c, i}-n_{r e c, i}-n_{\text {dea }, i}}{N_{i}} \times 100 \%
$$

$n_{\text {acc }, i}$ denoted the cumulative number of infections, $n_{\text {rec }, i}$ represented the cumulative number of recovered cases, $n_{\text {dea }, i}$ denoted the cumulative number of deaths, and $N_{i}$ was the population of country (or region) $i$.

The 194 countries were ranked by quintile into high risk, medium risk, and low risk regions. China has been selected as a representative country for the COVID-zero regions due to its ongoing zero-COVID-19 prevention and control strategy. The grouping result was shown in the Supplementary Table S1.

SUPPLEMENTARY TABLE S1. The detailed grouping results based on the prevalence of COVID-19 within 194 countries or regions.

\begin{tabular}{|c|c|c|c|c|c|}
\hline \multicolumn{2}{|c|}{ Low risk region } & \multicolumn{2}{|c|}{ Medium risk region } & \multicolumn{2}{|c|}{ High risk region } \\
\hline Country or region & $\begin{array}{c}\text { Prevalence } \\
(/ 100,000)\end{array}$ & Country or region & $\begin{array}{c}\text { Prevalence } \\
(/ 100,000)\end{array}$ & Country or region & $\begin{array}{c}\text { Prevalence } \\
(/ 100,000)\end{array}$ \\
\hline Chad & 0.14 & Dominican Republic & 91.30 & Iran & 495.50 \\
\hline Niger & 0.49 & Djibouti & 92.51 & Cuba & 521.52 \\
\hline Gambia & 0.54 & The Republic of Korea & 96.26 & Oman & 583.34 \\
\hline Senegal & 1.25 & Monaco & 96.83 & Ukraine & 606.80 \\
\hline Burkina Faso & 1.62 & Philippines & 98.45 & Turkey & 640.37 \\
\hline Bhutan & 2.20 & Spain & 105.77 & Lesotho & 651.28 \\
\hline Mali & 2.76 & Panama & 108.84 & Singapore & 661.37 \\
\hline Liberia & 2.79 & Congo & 116.33 & Bahamas & 663.96 \\
\hline Zambia & 3.67 & Czechia & 124.86 & North Macedonia & 691.11 \\
\hline Madagascar & 4.94 & Equatorial Guinea & 127.01 & Israel & 710.03 \\
\hline Nigeria & 5.17 & Hungary & 129.19 & Moldova & 710.32 \\
\hline Comoros & 5.63 & Central African Republic & 130.64 & Guyana & 710.94 \\
\hline Mozambique & 6.43 & Falkland Islands & 136.89 & Seychelles & 718.04 \\
\hline Kenya & 7.49 & Italy & 139.27 & Armenia & 760.45 \\
\hline Saudi Arabia & 7.57 & Cape Verde & 152.34 & Brunei & 795.00 \\
\hline Sudan & 8.53 & Nicaragua & 161.58 & Anguilla & 824.19 \\
\hline South Sudan & 9.44 & Greenland & 172.09 & Bulgaria & 873.81 \\
\hline Guinea & 9.70 & Iceland & 172.75 & Georgia & 883.76 \\
\hline Bangladesh & 10.16 & Montserrat & 172.98 & Romania & 904.68 \\
\hline Paraguay & 10.77 & Vietnam & 175.20 & Belize & 956.69 \\
\hline Ghana & 15.30 & Denmark & 179.53 & Latvia & 999.82 \\
\hline New Zealand & 17.52 & Algeria & 182.49 & Saint Lucia & $1,034.69$ \\
\hline
\end{tabular}


TABLE S1. (Continued)

\begin{tabular}{|c|c|c|c|c|c|}
\hline \multicolumn{2}{|c|}{ Low risk region } & \multicolumn{2}{|c|}{ Medium risk region } & \multicolumn{2}{|c|}{ High risk region } \\
\hline Country or region & $\begin{array}{l}\text { Prevalence } \\
(/ 100,000)\end{array}$ & Country or region & $\begin{array}{c}\text { Prevalence } \\
(/ 100,000)\end{array}$ & Country or region & $\begin{array}{c}\text { Prevalence } \\
(/ 100,000)\end{array}$ \\
\hline Mauritania & 20.28 & Jordan & 190.29 & Jamaica & $1,036.55$ \\
\hline Uzbekistan & 21.16 & San Marino & 191.53 & Barbados & $1,105.54$ \\
\hline India & 21.55 & Canada & 196.76 & $\begin{array}{l}\text { Bosnia and } \\
\text { Herzegovina }\end{array}$ & $1,107.93$ \\
\hline Malawi & 22.89 & Ecuador & 198.26 & Mauritius & $1,124.16$ \\
\hline Kuwait & 23.04 & Andorra & 199.31 & Libya & $1,152.96$ \\
\hline Sierra Leone & 23.08 & Bolivia & 203.64 & Dominica & $1,198.76$ \\
\hline Yemen & 24.84 & Brazil & 208.87 & Isle of Man & $1,228.95$ \\
\hline Pakistan & 26.07 & Turks and Caicos Islands & 209.21 & Estonia & $1,307.31$ \\
\hline Benin & 26.78 & Guernsey & 210.52 & Lithuania & $1,398.40$ \\
\hline $\begin{array}{l}\text { Democratic Republic of } \\
\text { Congo }\end{array}$ & 28.12 & Iraq & 212.04 & $\begin{array}{l}\text { Antigua and } \\
\text { Barbuda }\end{array}$ & $1,471.49$ \\
\hline Zimbabwe & 28.74 & Azerbaijan & 216.25 & Fiji & $1,497.08$ \\
\hline Ethiopia & 29.41 & Gabon & 217.73 & Palestine & $1,526.13$ \\
\hline Togo & 30.95 & Malta & 221.21 & Montenegro & $1,530.60$ \\
\hline Japan & 31.32 & Indonesia & 222.68 & Grenada & $1,748.15$ \\
\hline Egypt & 34.66 & Cayman Islands & 223.68 & Norway & $1,910.30$ \\
\hline Angola & 35.39 & Guatemala & 227.54 & Costa Rica & $1,938.34$ \\
\hline Guinea-Bissau & 37.75 & Sri Lanka & 238.31 & Finland & $2,053.73$ \\
\hline Papua New Guinea & 41.57 & Aruba & 243.52 & United Kingdom & $2,165.33$ \\
\hline Tanzania & 41.96 & Laos & 249.69 & $\begin{array}{l}\text { Saint Kitts and } \\
\text { Nevis }\end{array}$ & $2,286.48$ \\
\hline Morocco & 42.29 & Thailand & 251.62 & Mongolia & $2,335.06$ \\
\hline Rwanda & 42.91 & El Salvador & 269.31 & Honduras & $2,509.91$ \\
\hline Haiti & 44.14 & Botswana & 281.85 & Bermuda & $2,580.47$ \\
\hline Cote d'Ivoire & 48.33 & Belarus & 292.94 & Suriname & $2,714.13$ \\
\hline Kyrgyzstan & 48.53 & Mexico & 296.26 & Greece & $2,725.79$ \\
\hline Argentina & 50.25 & Germany & 310.60 & United States & $2,731.09$ \\
\hline Cameroon & 50.66 & Luxembourg & 312.52 & Slovakia & $2,832.00$ \\
\hline Cambodia & 53.11 & Portugal & 335.31 & New Caledonia & $3,168.48$ \\
\hline South Africa & 56.52 & Sao Tome and Principe & 365.03 & $\begin{array}{l}\text { Saint Vincent and } \\
\text { the Grenadines }\end{array}$ & $3,411.54$ \\
\hline Syria & 58.55 & Australia & 365.17 & Eswatini & $3,625.69$ \\
\hline Uruguay & 58.73 & Austria & 368.74 & Kazakhstan & $4,475.39$ \\
\hline Afghanistan & 60.30 & Liechtenstein & 374.96 & $\begin{array}{l}\text { Sint Maarten (Dutch } \\
\text { part) }\end{array}$ & $5,388.14$ \\
\hline Colombia & 60.32 & Faeroe Islands & 378.59 & Switzerland & $6,005.94$ \\
\hline Chile & 63.40 & Lebanon & 391.62 & Ireland & $7,350.34$ \\
\hline Qatar & 64.42 & Gibraltar & 391.80 & Curacao & $7,475.07$ \\
\hline Uganda & 65.72 & Jersey & 395.59 & French Polynesia & $8,281.83$ \\
\hline Namibia & 66.04 & Maldives & 396.27 & Cyprus & $9,804.61$ \\
\hline Somalia & 66.05 & Malaysia & 398.72 & France & $10,123.30$ \\
\hline United Arab Emirates & 67.92 & Trinidad and Tobago & 426.87 & Belgium & $10,503.50$ \\
\hline Bahrain & 68.35 & Albania & 437.07 & Sweden & $10,983.86$ \\
\hline Myanmar & 69.79 & British Virgin Islands & 439.86 & Netherlands & $11,607.85$ \\
\hline Nepal & 79.25 & Poland & 464.92 & Slovenia & $13,917.69$ \\
\hline Venezuela & 83.12 & Russia & 474.02 & Serbia & $14,227.67$ \\
\hline Tunisia & 86.69 & Croatia & 477.59 & & \\
\hline
\end{tabular}




\section{Model Formulation}

Considering that the effectiveness of COVID-19 vaccination was mainly focused on avoiding infection, avoiding symptomatic disease, and avoiding severe illness and death, this study extended the traditional epidemic model to include asymptomatic infection compartment and five corresponding compartments for vaccination. In addition, in order to assess the impact of population mobility on the spread of COVID-19, we assumed that high, medium, and low-risk regions would have a mobility rate of $R_{\text {trans }}$ with COVID-zero regions. To describe this flow, we randomly selected a population of size $R_{\text {trans }} \times N_{\text {clean }}$ from the total population of the clean region and a population of the same number from other regions separately to exchange. Using this method, we could realize the population mobility with the constant total population of each region.

Given the unknown safety of the vaccine in infants and young children, we assumed that this population would not be vaccinated. According to the population age distribution announced by China in 2020, infants aged 0-4 accounted for about $5 \%$ of the total population. Therefore, we assumed that the maximum proportion of vaccination was $95 \%$. This study focused on the epidemic trend of COVID-19 when a fairly high proportion of people have been vaccinated and international mobility resumed normal levels. Therefore, the starting of the prediction was that $95 \%$ of people have been vaccinated and no new vaccinations have taken place after all regions were totally opened.

\section{Model Equations}

$$
\begin{aligned}
& \frac{d S_{i}}{d t}=-\beta \times S_{i} \times \frac{\left[\left(I_{s y m, i}+I_{s y m, v, i}\right)+r_{a-s} \times\left(I_{a s y m, i}+I_{a s y m, v, i}\right)\right]}{N_{i}}-O u t_{S_{i}}+I n_{S_{i}} \\
& \frac{d S_{v, i}}{d t}=-\beta \times\left(1-e_{i n f}\right) \times S_{v, i} \times\left[\left(I_{s y m, i}+I_{s y m, v, i}\right)+r_{a-s} \times\left(I_{a s y m, i}+I_{a s y m, v, i}\right)\right] / N_{i}-O u t_{S_{v, i}}+\operatorname{In}_{S_{v, i}} \\
& \frac{d I_{s y m, i}}{d t}=r_{s y m} \times \beta \times r_{s y m} \times S_{i} \times\left[\left(I_{s y m, i}+I_{s y m, v, i}\right)+r_{a-s} \times\left(I_{a s y m, i}+I_{a s y m, v, i}\right)\right] / N_{i}-O u t_{I_{s y m, i}}+\operatorname{In}_{I_{\text {sym }, i}} \\
& \frac{d I_{\text {asym }, i}}{d t}=\left(1-r_{\text {sym }}\right) \times \beta \times r_{\text {sym }} \times S_{i} \times\left[\left(I_{\text {sym }, i}+I_{\text {sym }, v, i}\right)+r_{a-s} \times\left(I_{\text {asym }, i}+I_{\text {asym }, v, i}\right)\right] / N_{i}-O u t_{I_{\text {aym }, i}}+\operatorname{In}_{I_{\text {axym }, i}}
\end{aligned}
$$

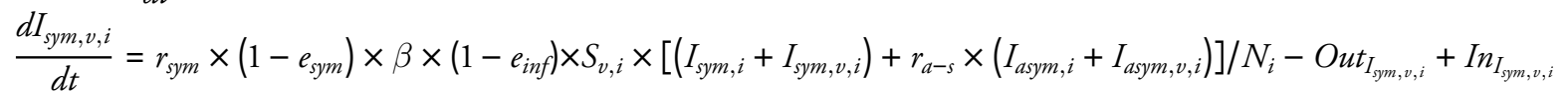

$$
\begin{aligned}
& \frac{d I_{\text {asym }, v, i}}{d t}=\left[1-r_{\text {sym }} \times\left(1-e_{\text {sym }}\right)\right] \times \beta \times\left(1-e_{\text {inf }}\right) \times S_{v, i} \times\left[\left(I_{\text {sym }, i}+I_{\text {sym }, v, i}\right)+r_{a-s} \times\left(I_{a s y m, i}+I_{\text {asym }, v, i}\right)\right] / N_{i}-O u t_{I_{a y m m}, v i}+\operatorname{In}_{I_{\text {aym }, v, i}} \\
& \frac{d R_{i}}{d t}=r_{r e c} \times\left(I_{s y m, i}+I_{\text {asym }, i}+I_{\text {sym }, v, i}+I_{\text {asym }, v, i}\right)-O u t_{R_{i}}+\operatorname{In}_{R_{i}} \\
& \frac{d D_{i}}{d t}=r_{\text {dea }} \times I_{\text {sym }, i}+\left(1-e_{\text {dea }}\right) \times r_{\text {dea }} \times I_{\text {sym }, v, i}
\end{aligned}
$$

Symbol $i$ included $0,1,2,3$ indicating clean, low risk, medium risk, and high-risk regions. We used the symbol $j$ to represent each compartment and the number of population mobility in different regions could be described by following equations.

For the COVID-zero Regions:

$$
\begin{gathered}
\text { Out }_{j, 0}=\sum_{1}^{3} R_{\text {trans }} \times N_{j, 0} \\
\operatorname{In}_{j, 0}=\sum_{i=1}^{3} \frac{R_{\text {trans }} \times N_{j, 0} \times N_{j, i}}{N_{i}}
\end{gathered}
$$

For the high risk, medium risk, and low risk regions $(i=1,2,3)$

$$
\begin{gathered}
O u t_{j, i}=\frac{R_{\text {trans }} \times N_{j, 0} \times N_{j, i}}{N_{i}} \\
\operatorname{In}_{j, 0}=R_{\text {trans }} \times N_{j, 0}
\end{gathered}
$$

\section{Parameter Estimation of $E_{\text {inf }}$}

At present, there were no definitive studies on the effectiveness of CoronaVac in preventing infection. After reviewing the literature, we found that in August 2021, Paul Elliott et al. (2) analyzed the efficacy against infection 
of COVID-19 vaccines administered in the UK. The conclusion was that the vaccine was $49 \%$ to $62 \%$ effective in preventing severe acute respiratory syndrome coronavirus 2 (SARS-CoV-2) infection in people aged 18 to 64 years old. Taking into account the higher efficacy of the Oxford/AstraZeneca COVID-19 vaccine in its published Phase III clinical data than CoronaVac, and the immune decline in the population after vaccination, we finally made an $\mathrm{E}_{\text {inf }}$ estimate of $30 \%$.

\section{REFERENCES}

1. Dingxiangyuan. Real time epidemic of New Coronavirus pneumonia 2020. 2021. https://ncov.dxy.cn/ncovh5/view/pneumonia. [2021-10-10]. (In Chinese).

2. U.S. Center for Disease Control and Prevention. COVID-19 pandemic planning scenarios. 2021. https://www.cdc.gov/coronavirus/2019-ncov/hcp/ planning-scenarios.html\#table-1. [2021-10-10]. 